\title{
Teacher education for Mathematical Literacy: A modelling approach
}

\author{
Bruce Brown and Marc Schäfer
}

Education Department, Rhodes University

B.Brown@ru.ac.za and M.Schafer@ru.ac.za

\section{Introduction}

The introduction of Mathematical Literacy into the Further Education and Training (FET) curriculum in South Africa has brought with it formidable challenges to teacher education in this field. This paper attempts to unravel some pertinent issues arising in the training of Mathematical Literacy teachers, using an approach based on mathematical modelling. It does this by discussing the design and implementation of an ACE(ML), an Advanced Certificate in Education, specialising in Mathematical Literacy teaching. A number of impressions were gained in the first implementation cycle of this course ending in December 2005. Lessons learned from these impressions were used to adapt and improve the course during delivery and will be used to further improve following cycles of the course. Specific impressions gained from two particular incidences in the course, together with a discussion on the theoretical underpinnings of the course form the core of this paper.

The programme under consideration was developed by the first author while working at Rhodes University Mathematics Education Project (RUMEP). It was developed in response to an urgent request by the Eastern Cape Department of Education, to provide professional training in Mathematical Literacy teaching, for 1000 teachers, for the implementation of the new FET curriculum in 2006.

A collaborative committee with representatives from all the higher education institutions in the Eastern Cape was constituted in order to facilitate this task. A curriculum subcommittee, chaired by the first author, developed the basic structure of the curriculum. This curriculum was accredited so that training of the first cohort could be started in the second half of 2004. Each participating higher educational institution in the Eastern Cape developed the detailed design of its own programme in line with the basic curriculum structure. The final programme implemented by
RUMEP for a cohort of 70 teachers is the focus of this paper.

\section{Mathematical Literacy}

The issue of what constitutes mathematical literacy is currently under debate. But a feature that does appear to distinguish mathematical literacy from mathematics is an emphasis on the use of mathematics in context (PISA 2003; Steen, 2001). In particular, contexts that are common or relevant to the day-to-day life of the 'ordinary' person in society (Duba, 2004; Laridon, 2004). And it is the use of mathematics in these contexts, by this 'ordinary' person that is an important focus of mathematical literacy. That is, to be mathematically literate, it is important that a person be able to identify mathematics relevant to the context at hand and then be able to use this mathematics as one means contributing towards the achievement of one's goals in the context.

One important observation that arose from this is that it may not be particularly helpful to view mathematical literacy as a global attribute of a person. It may be better to take a more local approach and view mathematical literacy as relative to context and so deal with mathematical literacy in context, for different contexts. A similar distinction is commonly made for literacy (PISA, 2003) and can be seen implicitly in the FET Curriculum documents (Department of Education, 2003) in their reference to the three broad contexts.

- Mathematical literacy for self-managing persons. The scope of this context is the use of mathematics for personal decision making and enrichment. A particularly important theme, that is identified as such in the Curriculum documents, is that of personal finances.

- Mathematical literacy for contributing workers. This encompasses the interpretation and use of mathematics common in the workplace. Particular examples of such mathematics are 
measurement, tables, schedules and graphs of productivity and performance.

- Mathematical literacy for participating citizens. Here the focus is on civic participation, involving the use of mathematics to engage with social issues and to interpret and respond to statements made by different actors in society. The ability to critically analyse statistics is often important in this context.

\section{Approach to teacher education}

We decided to adopt an activity- and investigationbased approach that is characterised by hands-on experience of a modelling process that seeks to develop an understanding of mathematics in context. Our course was broadly structured as follows:

- First, teachers themselves needed to develop positive experience of the use of mathematics in context, as described above. This experience of being mathematically literate themselves in some contexts, would enable them to appreciate the final goal for the subject, of enabling their learners to become mathematically literate in these contexts. Loosely, they may begin to know 'what it feels like' to be mathematically literate.

- Once teachers had developed their experience of mathematical literacy, they would reflect on the teaching and learning process that they had been through. In this way, they would be able to identify and conceptualise issues of teaching and learning important for the teaching of this subject. These issues would include practical matters such as planning, resources and assessment. They also included matters more difficult to formalise, such as processes of learning and how better to facilitate such processes.

- Teachers would also be asked to reflect on their experience of mathematical literacy 'in action' and so begin to develop their own, more formal, conception of what it means to be mathematically literate.

\section{A mathematical modelling approach to mathematical literacy}

When designing the teachers' training programme, an approach to the subject was sought that was aligned with mathematical literacy's particular focus on engaging with mathematics in ordinary contexts. In particular, we wished to encourage teachers to take an active view of mathematics, seeing it as relating naturally to life contexts and enabling them to make more effective decisions in such contexts. The process of identifying and then using relevant mathematics to analyse and make decisions about contexts showed many similarities to that of mathematical modelling. The major differences appeared to be that mathematical modelling is generally described using more advanced mathematics, in more technical contexts. But the basic approach of mathematical modelling could just as easily be applied to the use of elementary mathematics for modelling more everyday contexts (PISA, 2003).

The standard approach to mathematical modelling (Blum, 2002) may be very simply described as a cyclical process involving three components.

- Exploration of the context and formulation of the model and question.

- Analysis of the model in order to answer the mathematical question formulated.

- Interpretation and validation of the mathematical conclusion in terms of the context.

This description of the modelling process is based on the view that the person doing the modelling possesses the mathematical skills and concepts needed to formulate and analyse the model. But we expected the majority of the learners in the mathematical literacy class to display a rather low level of mathematical competence. As a result, they would need to work to develop mathematical skills and concepts as well as learning how to relate their growing mathematical understanding to contextual situations. With this in mind, we decided to adopt the "open modelling" approach to teaching described by Galbraith (1989) and Carr (1989). That is, learners should be encouraged to formulate and solve contextual problems using the mathematics that they find accessible. As a result, the mathematical model to be analysed could be anything from an instance of a simple numerical or spatial pattern, to an abstract mathematical system formulated in symbolic terms.

In order for teachers to build on this foundation and further develop learners' mathematical competence, we included a fourth component in the cycle.

- Consolidation and extension of the mathematical skills and concepts developed in the other stages of the cycle. 


\section{Bruce Brown and Marc Schäfer}

This would allow learners to develop mathematical understanding based on their experience of the grounding context and motivated by the use of the efficacy of the mathematics in context. This approach is similar to that taken in "Realistic Mathematics Education" advocated by the Freudenthal Institute (Van Den Heuwel-Panhuizen, 2000). They term the process of developing mathematical representations directly related to a grounding context "horizontal mathematicization". While building on this mathematical foundation to develop and refine further mathematical tools and representations is termed "vertical mathematicization".

With the above issues in mind, a more detailed conceptualisation of each stage of this modelling cycle was developed. This included a number of features that would not necessarily be considered an integral part of modelling, but would be important when using modelling as a vehicle for teaching Mathematical Literacy. This conceptualisation may be outlined as follows:

\section{Formulation:}

- Exploration of the context.

- Formulation of the model - identification and representation of measurable quantities and possible patterns in the context.

- Formulation of the question in the mathematical terms of the model.

- Identification and estimation of missing data.

Analysis of the model in order to answer the mathematical question formulated:

- Explore the regularities and relationships in the model.

- Develop mathematical tools to manipulate the model.

- Identify any extra assumptions which would make it possible to use the tools to answer the question.

- Use the tools developed to answer the mathematical question posed.

\section{Interpretation and validation of the mathematical conclusion in terms of the context:}

- Provide interpretations of mathematical representations and operations developed in the analysis.

- Interpret regularities and relationships developed within the analysis.
- Interpret the mathematical conclusion in terms of the context and the problem to be addressed in the context.

- Validate the interpreted conclusion by checking to see that it appears reasonable and in context and can be justified by appealing to the context.

- Understand the effects of the assumptions needed for the mathematical analysis, on the validity of the conclusions obtained.

- Understand the effects of the assumptions needed for the practical formulation of the model and posing of the question, on the validity of the conclusions obtained.

\section{Consolidation:}

- Practise using the mathematical representations and skills developed when formulating and analysing the model.

- Identify and explore mathematical relationships in the model.

- Identify and explore relationships between the mathematics developed to solve this problem and any other mathematical systems known by the modeller.

- Develop more abstract mathematical systems to represent and analyse these higher order relationships.

- Identify other contexts that may be related in similar ways to the model and mathematical systems developed in this process.

\section{Examples of the modelling approach}

Two tasks from the course will be discussed as examples of the teaching approach adopted. Both tasks were framed as assignments for a small business and so could be interpreted as falling in the broad context of "mathematics for contributing workers".

\section{a) Tiling a floor}

A simple example of the use of this modelling cycle in the course arose from the task of designing a tiled floor for a community hall. This task was initially framed as an assessment exercise for the course. Teachers were asked to design a pattern using black and white tiles, by first designing a pattern for a block of tiles and then replicating this block to cover the entire floor. They were provided with the dimensions of the hall ( $21 \mathrm{~m}$ by $15 \mathrm{~m})$, the 
dimensions of the block ( $3 \mathrm{~m}$ by $3 \mathrm{~m}$ ) and the dimensions of the tiles ( $300 \mathrm{~mm}$ by $300 \mathrm{~mm}$ ). For assessment, they were asked to draw the block they had designed (in a square outline provided), to count or calculate the number of each colour of tile in their block, and then to calculate the number of each colour of tile that would be needed to tile the community hall floor.

We had expected that most teachers would enjoy developing a creative design and would be able to calculate the number of tiles needed. Most teachers did indeed enjoy creating a design, but only half of the teachers on the course were able to calculate the number of tiles needed for the hall floor.

In response to this, a simple contextual problem involving tiling a floor (with a single tile type) was posed for group work later in the contact session.

- In the formulation stage, teachers were asked to explore and identify possible patterns in the context. In particular, to discuss the process of practically laying out tiles to cover the floor and to describe any patterns they could identify in this process that might be useful for counting the tiles. Most groups saw that they could approach the tiling task by laying out tiles in rows or columns across the floor. Some groups related this to the concept of area, but many did not.

- In the analysis stage, groups were asked to use the patterns they had identified to count the tiles and they were able to do this successfully. They developed different counting strategies to count the tiles, but at the end of the process, most groups had identified the product of the number of rows and the number of columns as important. Because the dimensions did not result in an integral number of rows and columns, different conclusions were reached, depending on how the remainders were handled.

- In the verification stage, the groups agreed on the importance of the basic product (rows $\times$ columns). This was followed by an animated discussion where groups described and justified their procedures for tiling the remaining space and described how these influenced their final estimate. At the end of the session the estimates of most groups had been accepted as reasonable. A few groups had used the concept of area to make their estimates. In justifying their results, they generally talked about the number of times the area of a tile could divide into the area of the floor. Some teachers were convinced by this, but others were sceptical.

- After this a short consolidation was carried out. The facilitator described the view of measuring area as covering a region with a unit area and then counting the number of units used to form the cover. The teachers were interested that this view of measuring area related the tiling situation directly to the area measurement process. Later in the course, a number of further consolidation tasks were carried out. These included calculating the area of a region by counting covering squares. They then spent time investigating how the measurement changed when the size of the unit square changed, and then practised doing this conversion in a number of cases.

Note that even though most of the teachers were able to solve the tiling problem using their different strategies, consolidation was necessary to enable them to better grasp the concept of area and develop related skills.

The reasoning of most groups was strongly dependent on, and expressed in terms of, the context of tiling that particular floor. In the subsequent discussion, these groups did state that the product of rows and columns was a useful and efficient summary, but they did not interpret this product in terms of area. When queried about area, they tended to define area as length times breadth, but they did not relate this to the product that had been developed for tiling. The solutions of these groups could thus be seen as situated in the context, as opposed to applications of an abstract mathematical concept (area) to the context (Collins, Brown and Duguid, 1989).

In the consolidation work, we worked from teachers' initial solutions to the problem to develop insight into ways that the concept of area, and the technical skills involved in calculating area, related to such tiling situations. These skills allowed them to solve similar tiling problems more efficiently in future. This was evidenced in their later assessment when most teachers were able to solve such tiling problems more efficiently using the mathematical skills that had been developed. 


\section{Bruce Brown and Marc Schäfer}

b) An investigation on designing parking lots One of the first investigations of the ACE(ML) was a group task that asked the teachers to design the parking spaces for cars in a parking lot. Teachers were provided with regulations for the minimum size of parking spaces of different orientations. They were also provided with a plan view showing the shape of the parking lot. The scale of the plan was given by specifying the actual width of the parking lot.

Achieving a fitting formulation for this problem was surprisingly problematical for most groups. Because of the openness of the task, it was expected that there would be a number of different responses. This was indeed the case. But what was surprising was that many of these differences were due to the teacher's view of the scale of the parking lot - a number of plans with different scales were drawn. Most groups spent very little time formulating the problem and identifying precisely what information they had. Instead they immediately began drawing a design.

This corresponds well to the behaviour of students who were faced with unfamiliar problems, reported by Schoenfeld (1992). Approximately $60 \%$ of these students made a quick decision and then pursued their chosen direction with no concurrent attempt to judge the success or failure of their attempt. In this case, it appeared as if the design was started without an explicit decision about the scale of the plan. The teachers seemed to make an implicit judgement of the scale and then proceeded using this implicit scale, disregarding the information that had been given about the length of the side of the lot.

The first attempt to correct the scales of the plans was unsuccessful. The facilitator asked these groups if a 'real' car would fit properly in their parking spaces. They all replied that it would, and demonstrated a car size that did fit their plan. In response, the facilitator demonstrated a car of a size consistent with the size of the parking lot given in the task, which was noticeably different from theirs. Most groups questioned the size of this car, rather than the scale of their plan.

The second attempt fared better. The facilitator asked the groups to calculate the scale of the plan by measuring the length of the side and comparing this with the specified length of that side. When asked to calculate the size of their parking spaces using this scale, the groups with very small parking spaces (less than half a metre wide) recognised a problem, but many of the other groups did not. But drawing a car to the calculated scale in one of their parking spaces, alerted the remaining groups to the inconsistency between their choice of scale and the size of the parking lot given in the task statement.

That the teachers were working relative to an implicit scale was evident from the fact that groups did not draw parking spaces of arbitrary sizes in their lots. In each design, the sizes of the parking places drawn were consistent. Possibly the teachers used a form of visual scaling, judging visually that the parking spaces were properly scaled according to a measure that they had intuitively chosen. In their response to the first question about the fit of a 'real' car, the inconsistent groups appeared to use the same visual scale for cars as they did for their parking lots, and so saw 'real' cars as indeed fitting their design.

Unfortunately, few groups used an intuitive scale that was fitting to the size of the lot specified in the task. Most groups saw the size of their parking lot as much larger, or much smaller than the size given in the task. When making their designs, these groups did not appear to interpret the given plan as a representation of a real parking lot. They appeared to understand that they were working on a 'problem' and not a design of a real parking lot and, as a consequence, they did not attend to the size of the parking lot given in the task.

In our work with the teachers, the interpretation of the plan as a representation of a real parking lot with dimensions as specified in the task, was crucial for the successful completion of the task. This interpretation could be seen as the process of relating mathematical objects or properties to objects and properties of a real situation. In this case:

- interpreting the mathematical object (the plan) in terms of a real object (the parking lot), and

- interpreting the dimensions of the plan as systematically related (by means of a fixed scale) to the corresponding dimensions of the real parking lot.

Even though this plan did not really relate to a real object, being prepared, and able, to make such interpretations would be important for using and drawing plans in life, where the plans would indeed relate to a real object.

The process of interpretation seemed to be made more difficult for the teachers by their understanding that this was a teaching task and so the first level of interpretation was artificial - in terms of a real object that did not exist. This may have made it easier to ignore the second level of interpretation needed. Such an issue could be a concern for the teaching of mathematical literacy, 
where most problem situations will not be real, even if they originate in real situations.

\section{Impressions gained}

\section{Context and content - the need for process}

Mathematical literacy involves the use of mathematics in context. To learn mathematical literacy, it is important to master the mathematics used, as well as to develop familiarity with the different contexts. But it is also necessary to develop the skills needed to be able to effectively relate mathematics and context. When training the teachers, we found it useful to explicitly identify such process skills, as these provided points of focus for identifying and developing these skills in their learners. As is evident in both examples, one such skill that was identified as important in teachers' responses was that of interpreting mathematical concepts and skills in relation to a context. A number of other important skills were identified in the training, including that of mathematical idealisation, or idealising contextual patterns and relations to form mathematical structures. This skill is discussed in the PISA framework (PISA 2003) as an important part of the modelling process. In the Mathematical Literacy training, we found it useful to view mathematical idealisation as dual to that of interpretation.

\section{The modelling approach}

a) Accessibility of the context based approach Most of the teachers appeared to enjoy this approach and found it quite accessible. As described in the example, they worked well in their groups and were able to develop effective solutions to the problems set, even if these were not very efficient. As well as familiarising themselves with the context, this work also provided a good foundation for the understanding and skills developed in the consolidation stage. Although initially most teachers elected to discuss the tasks between themselves, or with the facilitators, they were generally able to complete similar tasks successfully themselves.

\section{b) Time needed}

To successfully apply this teaching approach, the teachers on the course needed to be afforded time to effectively engage with the contextual problem. As an illustration of this, the episode described in the second example took place over a two and a half hour period. At the end of this period, most groups had developed a rough plan of their design using the correct scale - they were working in the analysis stage. As can be seen from the first example, properly completing such a contextual problem and then mastering the mathematical issues that arise in the process requires considerable time. But once done, these issues did appear to be well mastered with the mathematics learned being well related to the grounding question.

\section{c) Non-separateness of the modelling cycle}

It was noticed in facilitating these contextual problems that the components of the modelling cycle are not fully discrete. For the work at each stage was informed by the work done and the possibilities inherent in the other stages. That is, recognising patterns and developing the model was often guided as much by the possible mathematical structures known as it was by the characteristics of the context. Also, the analysis of the model was often guided by intuitive considerations based on the context. The modelling cycle thus became seen as a useful analytical tool, yielding insight into both the teaching and solution processes, rather than a constraining structure that needed to be rigidly followed.

\section{d) Need to balance contextualised work and consolidation}

Most of the teacher groups successfully completed the tiling problem introduced in response to the initial assessment in the first example. But many used a straightforward counting argument that, while simple and yielding a valid estimate, was rather inefficient, especially for large areas. Without the consolidation work, these groups would have probably continued to use this strategy in other problems of this type, particularly because they had successfully used it in the initial problem. The consolidation work enabled them to improve their mathematical understanding and to see how these skills could be used to more efficiently solve the contextual problem from which it flowed. In teaching the programme it became important to balance contextual work with consolidation.

\section{Conclusion}

The modelling approach worked well with the majority of reasonably and well skilled teachers. But many of the teachers with weaker mathematical skills took considerably longer to master the contexts and skills developed in the activities described above. This suggests that a teacher's level of mathematical skill is an important determinant of success in such a programme. But also that a lack of mathematical 


\section{Bruce Brown and Marc Schäfer}

skill is not the only barrier to success. Properly identifying these factors and their interrelationships would be an interesting question for further research.

Due to the pressure of time on the programme, it was not possible to include consolidation work that was appropriate for the less skilled teachers, for all the contexts considered. The first objective of providing teachers with the experience of being properly mathematically literate, was thus not fully achieved for less skilled teachers. In consequence, the more reflective parts of the programme became less real to them and were partly memorised rather than evidenced through experience.

\section{References}

Blum, W. (2002). ICMI study 14: Applications and modelling in mathematics education. Discussion document. Educational Studies in Mathematics 51, 149-171.

Carr, A. (1989). Teaching mathematical modelling. In D. Blane \& M. Evans (Eds.), Mathematical modelling for the senior years (pp. 66-71). Parkville: The Mathematical Association of Victoria.

Collins, A., Brown, J.S.A., and Duguid, P. (1989). Situated cognition and the culture of learning. Educational Researcher, 18(1), 32-42.

Department of Education. (2003). National Curriculum Statement Grades 10-12 (General), Mathematical Literacy. Pretoria: Government Printer.
Duba, T. (2004). Mathematical Literacy: An International Perspective. Paper presented at the Shuttleworth Mathematical Literacy Seminar, Cape Town, February 2004.

Galbraith, P. (1989). From applications to modelling. In D. Blane \& M. Evans (Eds.), Mathematical modelling for the senior years (pp. 78-86). Parkville: The Mathematical Association of Victoria.

Laridon, P. (2004). Mathematical Literacy and the FET Curriculum. Paper presented at the Shuttleworth Mathematical Literacy Seminar, Cape Town, February 2004.

PISA. (2003). Assessment Framework: Mathematics, Reading, Science and Problem Solving Knowledge and Skills - Publications 2003.

Schoenfeld, A.H. (1992). Learning to think mathematically: Problem solving, metacognition and sense making in mathematics. In D. Grouws (Ed.), Handbook of research on mathematics teaching and learning (pp. 334-370). New York: Macmillan.

Steen, L. (2001). The Case for Quantitative Literacy. In L. A. Steen (Ed.), Mathematics and Democracy: The Case for Quantitative Literacy (pp. 1-22). USA: National Council on Education and the Disciplines.

Van Den Heuwel-Panhuizen, M. (2000). Mathematics education in the Netherlands: A guided tour. Freudenthal Institute CD-rom for ICME9. Utrecht: Utrecht University.

\section{Errors using inadequate data are much less than those}

using no data at all.

- Charles Babbage 